\title{
High-Cooperativity Coupling of a Rare-Earth Spin Ensemble to a Superconducting Resonator Using Yttrium Orthosilicate as a Substrate
}

\author{
Gavin Dold,,${ }^{1,2,{ }^{*}}$ Christoph W. Zollitsch, ${ }^{1}$ James O’Sullivan, ${ }^{1}$ Sacha Welinski, ${ }^{3}$ Alban Ferrier, ${ }^{3,4}$ \\ Philippe Goldner, ${ }^{3}$ S.E. de Graaf, ${ }^{2}$ Tobias Lindström, ${ }^{2}$ and John J.L. Morton ${ }^{1,5, \dagger}$ \\ ${ }^{1}$ London Centre for Nanotechnology, University College London, London WC1H 0AH, United Kingdom \\ ${ }^{2}$ National Physical Laboratory, Hampton Road, Teddington TW11 0LW, United Kingdom \\ ${ }^{3}$ Université PSL, Chimie ParisTech, CNRS, Institut de Recherche de Chimie Paris, 75005 Paris, France \\ ${ }^{4}$ Faculté des Sciences et Ingénierie, Sorbonne Université, 75005 Paris, France \\ ${ }^{5}$ Department of Electronic and Electrical Engineering, UCL, London WC1E 7JE, United Kingdom
}

(Received 6 December 2018; revised manuscript received 10 April 2019; published 29 May 2019)

\begin{abstract}
Yttrium orthosilicate $\left(\mathrm{Y}_{2} \mathrm{SiO}_{5}\right.$, or $\left.\mathrm{YSO}\right)$ has proved to be a convenient host for rare-earth ions used in demonstrations of microwave quantum memories and optical memories with microwave interfaces and shows promise for coherent microwave-optical conversion owing to its favorable optical and spin properties. The strong coupling required by such microwave applications could be achieved using superconducting resonators patterned directly on $\mathrm{Y}_{2} \mathrm{SiO}_{5}$ and hence we investigate here the use of $\mathrm{Y}_{2} \mathrm{SiO}_{5}$ as an alternative to sapphire or silicon substrates for superconducting-hybrid-device fabrication. A NbN resonator with frequency $6.008 \mathrm{GHz}$ and low-power quality factor $Q \approx 400000$ is fabricated on a $\mathrm{Y}_{2} \mathrm{SiO}_{5}$ substrate doped with isotopically enriched ${ }^{145} \mathrm{Nd}$. Measurements of dielectric loss yield a loss tangent $\tan \delta=4 \times 10^{-6}$, comparable to that of sapphire. Electron spin resonance (ESR) measurements performed using the resonator show the characteristic angular dependence expected from the anisotropic ${ }^{145} \mathrm{Nd}$ spin and the coupling strength between the resonator and the electron spins is in the high-cooperativity regime $(C=30)$. These results demonstrate that $\mathrm{Y}_{2} \mathrm{SiO}_{5}$ is an excellent substrate for low-loss, high- $Q$ microwave resonators, especially in applications for coupling to optically accessible rare-earth spins.
\end{abstract}

DOI: 10.1103/PhysRevApplied.11.054082

\section{INTRODUCTION}

Rare-earth ions (REIs) in crystals are promising candidate systems for quantum-information applications, as they possess electron and nuclear spins as well as optical transitions at telecom wavelengths. Coherence times in these systems range from milliseconds [1] for microwave excitations stored in an electron spin to hours [2,3] for optically accessible nuclear excitations. REIs typically possess large $g$ factors (beneficial for strong coupling), have a variety of nuclear spin states, and offer opportunities for optical pumping. Such properties have led to proposals for using rare-earth ions as a multimode microwave quantum memory [4], a photonic memory for a quantum repeater [5], and as a microwave-optical transducer $[6,7]$ for use in quantum networks [8].

Yttrium orthosilicate $\left(\mathrm{Y}_{2} \mathrm{SiO}_{5}\right)$ is a widely used crystalline host for REIs in such quantum-information applications, as its constituent elements provide an environment with a low background of nuclear magnetic moments

*gavin.dold.15@ucl.ac.uk

†jjl.morton@ucl.ac.uk that would otherwise contribute to spin decoherence and inhomogeneous broadening. The narrow homogeneous linewidths - for example, down to $3.5 \mathrm{kHz}$ for the 883 $\mathrm{nm}$ transition [9] in $\mathrm{Nd}^{3+}: \mathrm{Y}_{2} \mathrm{SiO}_{5}$ - have been exploited as an optical quantum memory [10,11] enabling storage of entangled states [12] and teleportation with $93 \%$ fidelity [13].

While optical-storage experiments using REIs in $\mathrm{Y}_{2} \mathrm{SiO}_{5}$ can make use of a suitably optically dense medium, for microwave storage a cavity is employed to achieve a coupling between an ensemble of REIs and the microwave-cavity field, so that excitations can be coherently exchanged between the two. This can be achieved with three-dimensional (3D) microwave cavities [7], which offer homogeneous $B_{1}$ fields and spatial mode matching between microwave and optical fields, or planar superconducting resonators with small mode volumes and quality factors over $10^{5}$, to yield high spin-number sensitivities [14] and act as an interface between superconducting qubits and spin ensembles [15]. Dielectric resonators fabricated from rare-earth-doped crystals such as YAG $\left(\mathrm{Y}_{3} \mathrm{Al}_{5} \mathrm{O}_{12}\right)$ [16] have used resonant modes with $Q \approx 10^{4}$ to drive ESR transitions with cooperativity $C \approx 600$, while 
planar superconducting resonators have been coupled to rare earths $\left(Q \approx 10^{3}, C=36\right.$, [17]), group V donors in silicon $[14,18]$, nitrogen-vacancy $(\mathrm{NV})$ centers in diamond [15], and ruby $\left(\mathrm{Al}_{2} \mathrm{O}_{3}: \mathrm{Cr}^{3+}\right)$ [19].

Experiments coupling superconducting resonators to doped crystals have typically used a flip-chip approach, with the sample glued or mechanically pressed onto the resonator chip [17]. This has the drawback of complicating the fabrication process and creates an additional interface layer between the resonator and sample, increasing dielectric losses from spurious two-level systems (TLSs) in the interface [20], as well as a variable gap between the device and the spins, which is less controllable than fabrication on the substrate itself.

Common substrates for superconducting resonators include silicon and sapphire $\left(\mathrm{Al}_{2} \mathrm{O}_{3}\right)$ [21]. Dielectric losses in these are usually quantified by the loss tangent $\tan \delta$, which describes the ratio between the imaginary and real components of the complex permittivity of the material. This creates a limit on the quality factor achievable in superconducting resonators: $Q=1 / \tan \delta$. For high$Q$ devices, sapphire is often preferred due to its low loss tangent [22] $\tan \delta<10^{-5}$, leading to quality factors approaching $10^{6}$.

In this work, we investigate the suitability of using $\mathrm{Y}_{2} \mathrm{SiO}_{5}$ itself as a substrate for fabrication of planar superconducting devices. We fabricate a resonator on the polished surface of a Nd-doped $\mathrm{Y}_{2} \mathrm{SiO}_{5}$ sample and find that the device has a $Q$ factor $>10^{5}$ at low powers due to its low loss tangent $\tan \delta=4 \times 10^{-6}$. We observe an anisotropic ESR spectrum matching that from simulations and measure the coupling strength between the resonator and the electron spin to be in the high-cooperativity regime. These results suggest that fabrication on doped $\mathrm{Y}_{2} \mathrm{SiO}_{5}$ is compatible with high- $Q$ devices while simultaneously enabling a coupling to crystals doped with rareearth ions and could be a promising route toward scalable hybrid superconductor-spin quantum circuits.

\section{THE DEVICE}

The device is a lumped-element superconducting resonator, fabricated on a Czochralski-grown single crystal of $\mathrm{Y}_{2} \mathrm{SiO}_{5}$ [23] doped with $10 \mathrm{ppm}$ isotopically purified ${ }^{145} \mathrm{Nd}$. The crystal is cut into a $5 \mathrm{~mm} \times 5 \mathrm{~mm} \times 460 \mu \mathrm{m}$ chip along the principal dielectric axes $\left(D_{1}, D_{2}\right.$, and $\left.b\right)$ and a face perpendicular to $b$ is polished for thin-film growth. $\mathrm{Y}_{2} \mathrm{SiO}_{5}$ has two inequivalent crystal sites where a $\mathrm{Y}^{3+}$ ion can be substituted by a rare-earth $\mathrm{RE}^{3+}$ ion. The large ionic radius of $\mathrm{Nd}^{3+}$ results in the larger crystal Site 1 being preferentially populated [1], resulting in a stronger signal from site 1 over site 2 . Due to the crystal's $C_{2 h}^{6}(C 2 / c)$ space group, each site has two orientations related by a $\pi$ rotation around the crystal $b$ axis. These two orientations are termed subsites and their ESR properties are degenerate (a)
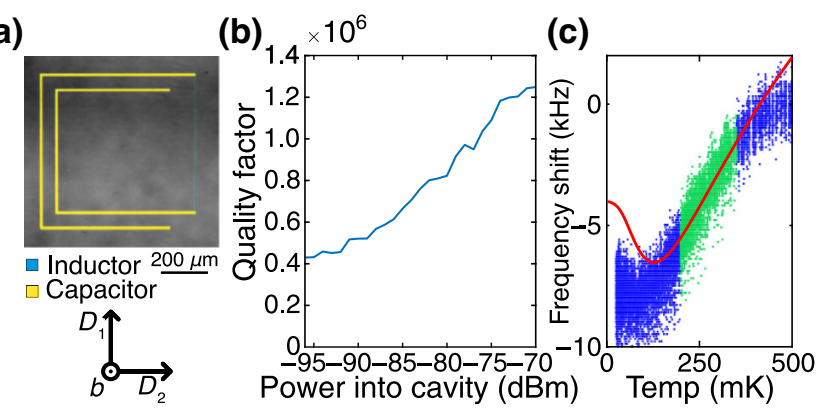

FIG. 1. (a) A false-color micrograph of the superconducting thin-ring resonator on ${ }^{145} \mathrm{Nd}: \mathrm{Y}_{2} \mathrm{SiO}_{5}$ substrate. (b) The power dependence of the loaded quality factor at zero field. At high powers, the TLSs begin to saturate, resulting in an increase in $Q$ [24]. (c) The frequency shift of the resonator as a function of the temperature, from which a fit to theory [22] (red) of the gradient of the linear section (green) yields the loss tangent $\tan \delta=4 \times 10^{-6}$.

for $B_{0}$ fields applied in the $D_{1}-D_{2}$ plane or parallel to the $b$ axis.

The resonator consists of a $2 \mu \mathrm{m}$ wire, which functions as an inductor due to the constriction increasing the contribution of kinetic inductance, and a pair of $10-\mu \mathrm{m}$-thick capacitive arms separated by a $50-\mu \mathrm{m}$ gap. The use of narrow features increases the resilience of the resonator to the applied magnetic field and the large gap between the capacitive arms reduces the peak electric field of the resonator, decreasing its susceptibility to dielectric losses from two-level systems. This forms a lumped-element resonator, seen in the micrograph in Fig. 1, which generates an oscillating $B_{1}$ magnetic field around the inductor that can drive ESR transitions. Details of this resonator design's properties in an applied magnetic field will be described in a future publication.

Fabrication consists of $40 \mathrm{~nm}$ of sputtered $\mathrm{NbN}$ being patterned by photolithography and a $\mathrm{SF}_{6} /$ Ar reactive-ionetch process. The patterned chip is enclosed within a $3 \mathrm{D}$ copper cavity $(Q \approx 100)$ to suppress spontaneous emission from the resonator to the environment [14]. This is installed in the bore of a vector magnet in a dilution refrigerator at $10 \mathrm{mK}$ (measured at the mixing chamber plate) and probed using a vector network analyser (VNA) at zero applied magnetic field.

The lumped-element resonator has a frequency of 6.008 $\mathrm{GHz}$ and an asymmetric lineshape due to interference with the background transmission of the 3D cavity. Fitting with a Fano resonance [25] yields a quality factor $Q \approx 400000$ in the low-power limit in Fig. 1. By tracking the center frequency versus the temperature [24], we find that the dielectric losses in the device are comparable to those of resonators fabricated on sapphire [22,26], with a loss tangent $\tan \delta=4 \times 10^{-6}$, where the filling factor $F \approx \frac{1}{2}$ representing the fraction of the magnetic field penetrating the substrate has been factored out. 
This demonstrates that $\mathrm{Y}_{2} \mathrm{SiO}_{5}$ is well suited for devices incorporating resonators and superconducting qubits, which are typically susceptible to dielectric losses from TLSs [27], while also incorporating doped spins for cavity quantum electrodynamics (QED).

\section{ELECTRON SPIN RESONANCE}

\section{A. ESR spectrum}

Having studied the resonator, we now use it to perform ESR to characterize the $\mathrm{Nd}$ spins in the $\mathrm{Y}_{2} \mathrm{SiO}_{5}$ that are coupled to the resonator. A magnetic field $B_{0}$ is applied in the plane of the superconducting thin film, roughly perpendicular to the $\mathrm{Y}_{2} \mathrm{SiO}_{5}$ crystal $b$ axis, to minimize the magnetic flux threaded through the superconductor. The field is swept up to $360 \mathrm{mT}$ and the resonator is used to observe ESR transitions, monitoring changes to its center frequency tracked with a VNA. By this method, a series of spectra are taken as a function of the $B_{0}$ orientation in the $D_{1}-D_{2}$ plane to extract the angular dependence, or "roadmap", of ESR line positions, in order to confirm the presence of $\mathrm{Nd}$ spins in the substrate and measure their coupling to the resonator.

For an electron spin $\mathbf{S}$ coupled to a nuclear spin $\mathbf{I}$, the Hamiltonian accounting for the electron Zeeman and hyperfine terms is $H=\mu_{B} \mathbf{B}^{\mathbf{T}} \mathbf{g} \hat{\mathbf{S}}+\hat{\mathbf{S}}^{\mathbf{T}} \mathbf{A} \hat{\mathbf{I}}$. The anisotropy of $\mathrm{Y}_{2} \mathrm{SiO}_{5}$ results in the parameters $\mathrm{g}$ and $\mathrm{A}$ being tensors, previously calculated from spectroscopic studies of Site 1 of ${ }^{145} \mathrm{Nd}: \mathrm{Y}_{2} \mathrm{SiO}_{5}$ at $9.4 \mathrm{GHz}$ [28] as follows:

$$
\begin{aligned}
g & =\left(\begin{array}{ccc}
1.30 & 0.62 & 0.22 \\
0.62 & -2.07 & 1.62 \\
0.22 & 1.62 & -2.86
\end{array}\right)_{\left(D_{1}, D_{2}, b\right)}, \\
A & =\left(\begin{array}{ccc}
-37.1 & -99.9 & -83.4 \\
-99.9 & -589.2 & 169.4 \\
-83.4 & 169.4 & -678.4
\end{array}\right)_{\left(D_{1}, D_{2}, b\right)} \text { MHz. }
\end{aligned}
$$

A characteristic ESR spectrum ( $B_{0}$ along the $D_{1}$ axis) is plotted in Fig. 2(b). We observe a series of eight ESR transitions indicated in Fig. 2(a), corresponding to the ${ }^{145} \mathrm{Nd}$ nuclear $\operatorname{spin}\left(I=\frac{7}{2}\right)$ in Site 1 . A small misalignment between the $D_{1}-D_{2}$ and the superconductor planes breaks the degeneracy between the crystal subsites and causes a further splitting of each line into two.

The roadmap of the ESR spectrum with respect to the $B_{0}$-field angle is plotted in Fig. 2(c), with transitions marked by circles. The angular dependence seen in these spectra matches simulations well for $\mathrm{Nd}$ spins in Site 1 (blue), while we also observe a signal at lower magnetic fields (higher $g$ factor), which we attribute to Site 2 (red). The full spin Hamiltonian of ${ }^{145} \mathrm{Nd}$ in Site 2, including hyperfine terms, has not been reported; therefore our simulations only account for the Zeeman term (i.e., the $g$ tensor). Additional unidentified impurities are evident from the different angular dependencies of 2-3 transitions marked in green and black.
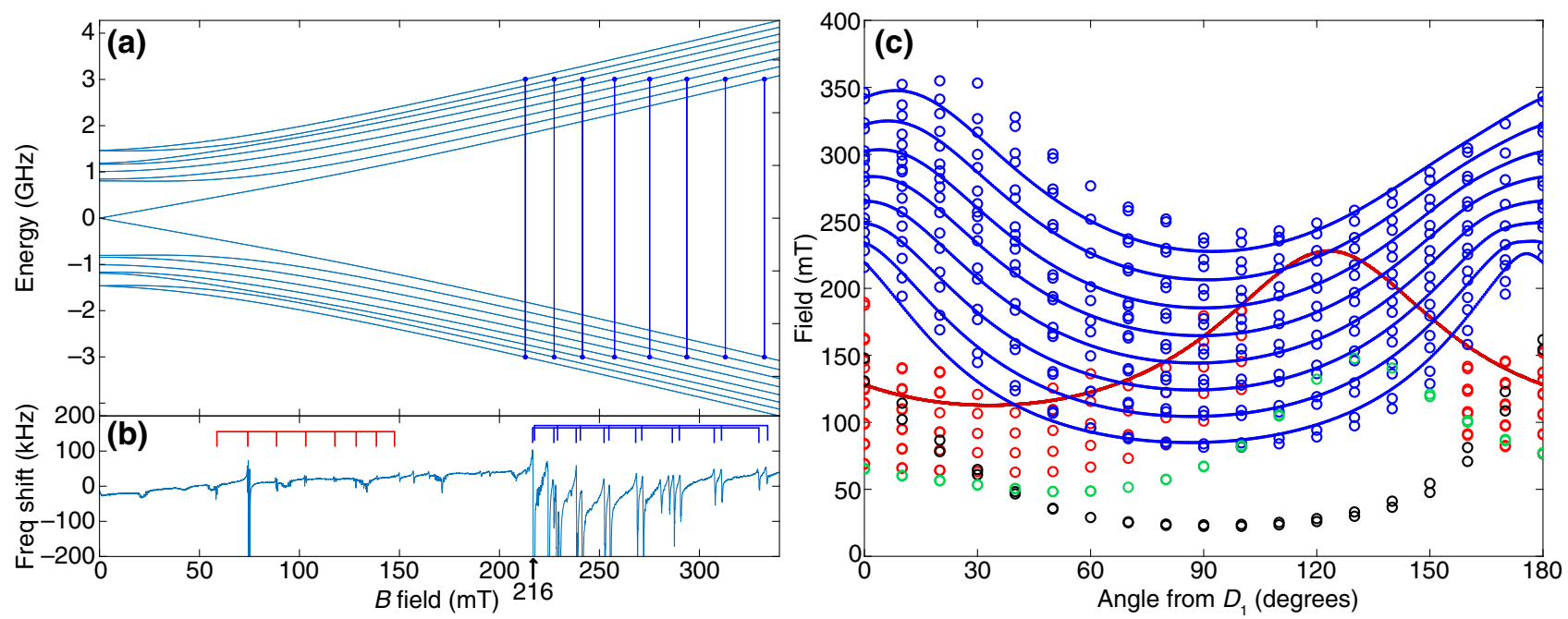

FIG. 2. (a) A Breit-Rabi diagram of ${ }^{145} \mathrm{Nd}$ in $\mathrm{Y}_{2} \mathrm{SiO}_{5}$ for magnetic field $B_{0}$ oriented along $D_{1}$, with ESR transitions at $6.008 \mathrm{GHz}$ indicated. (b) The ESR spectrum with the magnetic field $B_{0}$ oriented along $D_{1}$, as measured with a VNA tracking the resonator center frequency. The quadratic dependence of the resonator frequency with the applied magnetic field has been subtracted. Transitions from Site 1 are indicated in blue and those from Site 2 in red. Two sets of Site- 1 transitions are seen due to a broken subsite degeneracy. The avoided crossing studied in Sec. III B is indicated at $B_{0}=216 \mathrm{mT}$. (c) The roadmap of ESR transition fields with respect to the $B_{0}$ angle from the $D_{1}$ axis, in the $D_{1}-D_{2}$ plane. The circles mark the positions of transitions from traces such as in (b), with Site 1 in blue and Site 2 in red. Signals from Site 2 between $110^{\circ}$ and $150^{\circ}$ could not be resolved due to weak transition amplitudes at these angles. The lines represent simulated data assuming an ideal rotation about the crystal $b$ axis. For Site 2 , we only show one line, as only the $g$ tensor is known for this site. The green and black colors represent as-yet-unidentified impurities. 
These measurements enable ESR transitions to be positively identified by comparing their angular dependence to simulations [29] and bulk ESR data, ensuring that further analysis of spin-resonator coupling properties can be clearly linked to a particular spin species.

\section{B. High-cooperativity coupling}

The highest-intensity ESR transition for ${ }^{145} \mathrm{Nd}$ in Site 1 with $m_{I}=\frac{7}{2}$ at $B_{0}=216 \mathrm{mT}$ along $D_{1}$ is selected for an evaluation of the spin-resonator coupling strength. Measuring the resonator $S_{21}$ with a VNA while sweeping the magnetic field strength $B$, we observe the onset of an avoided crossing shown in Fig. 3. Fitting of the resonator frequency $\omega$ and linewidth $\kappa$ at each field point [30] allows us to extract the coupling strength $g_{\text {ens }}$, the inhomogeneous spin-ensemble half-width $\gamma_{s}$, and the resonator half-width $\kappa_{c}$ with

$$
\begin{aligned}
& \omega=\omega_{c}-g_{\text {ens }}^{2} \Delta /\left(\Delta^{2}+\gamma_{s}^{2}\right), \\
& \kappa=\kappa_{c}-g_{\text {ens }}^{2} \gamma_{s} /\left(\Delta^{2}+\gamma_{s}^{2}\right),
\end{aligned}
$$

where $\Delta=m_{0}\left(B-B_{0}\right) / \hbar$ is the field detuning calculated from the spin magnetic moment $m_{0}=\hbar\left(d \omega / d B_{0}\right)$. This accounts for cases where $d \omega / d B$ varies with $B$, as is the
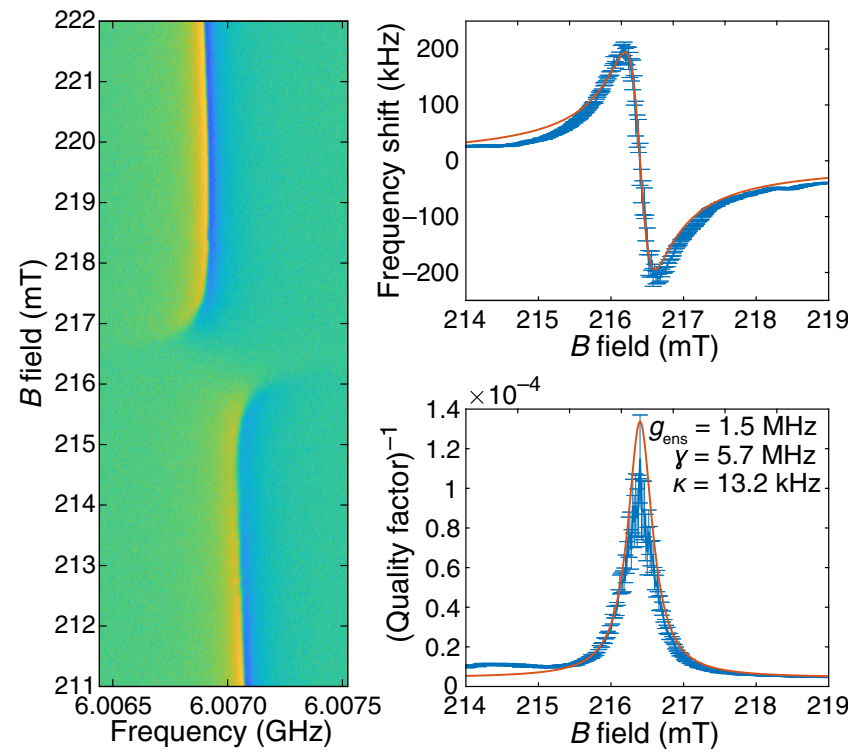

FIG. 3. Left: the onset of an avoided crossing when sweeping the $B$ field through resonance. Right: the resonator frequency shift and quality factor as a function of the $B$-field strength when sweeping through resonance. The uncertainty in the resonator quality factor increases at the center of the transition due to a reduction in prominence of the resonance. A simultaneous fit to all resonances as a function of the magnetic field, using the equations in the main text, yields a dependence of the frequency and the quality factor with the field shown in red and a high-cooperativity coupling $C=30$. case for mixed-spin systems in the low-field limit or near zero first-order Zeeman (ZEFOZ) points.

From this, we extract $g_{\text {ens }}=1.5 \mathrm{MHz}, \gamma_{s}=5.7 \mathrm{MHz}$, and $\kappa_{c}=13.2 \mathrm{kHz}$. This corresponds to a high cooperativity $C=g_{\text {ens }}^{2} / \kappa_{c} \gamma_{s}=30$.

For a bulk-doped sample, there is no clear single-spin coupling rate, as the $1 / r$ decay of the field strength from the wire results in a $B_{1}$ inhomogeneity spanning orders of magnitude, while all spins in the sample contribute to the signal. However, a characteristic rate representing the average coupling strength over the spins contributing $50 \%$ of the measured signal can be calculated. This corresponds to a region within $4 \mu \mathrm{m}$ of the wire with $N \approx 6 \times 10^{8}$ resonant ${ }^{145} \mathrm{Nd}$ spins in the same subsite, accounting for the thermal population in the $m_{I}=\frac{7}{2}$ ESR transition at 10 $\mathrm{mK}$. From this, we derive a characteristic single-spin coupling rate $g_{0}=g_{\text {ens }} / \sqrt{N} \approx 50 \mathrm{~Hz}$, leading to an expected Purcell enhanced emission rate [31] of $\Gamma_{\mathrm{P}}=4 g_{0}^{2} / \kappa_{c} \approx$ $1 \mathrm{~Hz}$.

These results indicate that the coupling regime is limited by the spin line width $\gamma_{s}>g_{\text {ens }}$ or, equivalently, that the $\mathrm{Nd}$ spin ensemble is decohering at a faster rate than excitations are being exchanged between it and the resonator. Steps toward improving this coupling strength could include using a more strongly doped rare-earth sample to increase $g_{\text {ens }}$, although this could also increase $\gamma_{s}$, or exploiting the coherence-enhancing ZEFOZ transitions in REIs [32] to decrease $\gamma_{s}$. The significant $B_{1}$ inhomogeneity in this resonator design also poses challenges for performing coherent operations on the entire spin ensemble with pulsed ESR. This could be mitigated by coupling to implanted layers of REIs $[29,33]$ or by using resonators designed to generate a more homogeneous $B_{1}$ field.

\section{CONCLUSION}

We investigate the use of $\mathrm{Y}_{2} \mathrm{SiO}_{5}$ as an alternative to sapphire or silicon substrates for the fabrication of superconducting devices. The fabricated $\mathrm{NbN}$ lumped-element resonator has a quality factor $Q \approx 400000$ and a loss tangent $\tan \delta=4 \times 10^{-6}$ comparable to that of sapphire [22] and yields a high-cooperativity coupling $C=30$ between the resonator and a ${ }^{145} \mathrm{Nd}$ spin ensemble. These results demonstrate that $\mathrm{Y}_{2} \mathrm{SiO}_{5}$ is well suited for superconducting devices, while also enabling an interaction with optically accessible rare-earth spins.

Further studies of this device include performing pulsed ESR measurements in the high-cooperativity regime and measuring the coherence properties of the spin ensemble. The methods shown here are also applicable to other REIs in $\mathrm{Y}_{2} \mathrm{SiO}_{5}$; for example, $\mathrm{Er}$, with its 1540-nm telecomband optical transition [7], and $\mathrm{Yb}$, which exhibits a large oscillator strength [34] and coherence-enhancing ZEFOZ 
and near-ZEFOZ transitions [32]. It may also prove interesting to study the suitability as a substrate of other crystalline hosts for REIs, such as yttrium aluminium garnet (YAG), yttrium lithium fluoride (YLF), yttrium orthovanadate $\left(\mathrm{YVO}_{4}\right)$, and calcium tungstate $\left(\mathrm{CaWO}_{4}\right)$.

The fabrication of superconducting devices on crystals doped with REIs as demonstrated here shows promise for integrating these optical elements alongside the fast information processing available from superconducting qubits. This is an important step toward making a microwaveoptical transducer capable of connecting such quantum processors within a quantum network. By exploiting the long coherence times available from REIs, this is also a route to integrating fast quantum electronics with a spin ensemble acting as a quantum memory.

\section{ACKNOWLEDGMENTS}

This work has received funding from the Engineering and Physical Sciences Research Council (EPSRC) through the Centre for Doctoral Training in Delivering Quantum Technologies (Grant No. EP/L015242/1), the European Community's Seventh Framework Programme Grant No. 279781 (ASCENT), the IMTO Cancer AVIESAN (Cancer Plan, C16027HS, MALT), and the United Kingdom government's Department for Business, Energy and Industrial Strategy.

[1] G. Wolfowicz, H. Maier-Flaig, R. Marino, A. Ferrier, H. Vezin, J. J. L. Morton, and P. Goldner, Coherent Storage of Microwave Excitations in Rare-Earth Nuclear Spins, Phys. Rev. Lett. 114, 170503 (2015).

[2] M. Zhong, M. P. Hedges, R. L. Ahlefeldt, J. G. Bartholomew, S. E. Beavan, S. M. Wittig, J. J. Longdell, and M. J. Sellars, Optically addressable nuclear spins in a solid with a six-hour coherence time, Nature 517, 177 (2015).

[3] M. Rančić, M. P. Hedges, R. L. Ahlefeldt, and M. J. Sellars, Coherence time of over a second in a telecomcompatible quantum memory storage material, Nat. Phys. 14, 50 (2017).

[4] S. Probst, H. Rotzinger, A. V. Ustinov, and P. A. Bushev, Microwave multimode memory with an erbium spin ensemble, Phys. Rev. B 92, 014421 (2015).

[5] N. Sangouard, C. Simon, H. de Riedmatten, and N. Gisin, Quantum repeaters based on atomic ensembles and linear optics, Rev. Mod. Phys. 83, 33 (2011).

[6] L. A. Williamson, Y. H. Chen, and J. J. Longdell, MagnetoOptic Modulator with Unit Quantum Efficiency, Phys. Rev. Lett. 113, 1 (2014).

[7] X. Fernandez-Gonzalvo, Y. H. Chen, C. Yin, S. Rogge, and J. J. Longdell, Coherent frequency up-conversion of microwaves to the optical telecommunications band in an Er:YSO crystal, Phys. Rev. A—Atomic Mol. Opt. Phys. 92, 1 (2015).
[8] H. J. Kimble, The quantum internet, Nature 453, 1023 (2008).

[9] I. Usmani, M. Afzelius, H. de Riedmatten, and N. Gisin, Mapping multiple photonic qubits into and out of one solidstate atomic ensemble, Nat. Commun. 1, 1 (2010).

[10] M. P. Hedges, J. J. Longdell, Y. Li, and M. J. Sellars, Efficient quantum memory for light, Nature 465, 1052 (2010).

[11] P. Jobez, C. Laplane, N. Timoney, N. Gisin, A. Ferrier, P. Goldner, and M. Afzelius, Coherent Spin Control at the Quantum Level in an Ensemble-Based Optical Memory, Phys. Rev. Lett. 114, 230502 (2015).

[12] C. Clausen, I. Usmani, F. Bussières, N. Sangouard, M. Afzelius, H. de Riedmatten, and N. Gisin, Quantum storage of photonic entanglement in a crystal, Nature 469, 508 (2011).

[13] F. Bussieres, C. Clausen, A. Tiranov, B. Korzh, V. B. Verma, S. W. Nam, F. Marsili, A. Ferrier, P. Goldner, H. Herrmann, C. Silberhorn, W. Sohler, M. Afzelius, and N. Gisin, Quantum teleportation from a telecom-wavelength photon to a solid-state quantum memory, Nat. Photonics 8 , 775 (2014).

[14] A. Bienfait, J. J. Pla, Y. Kubo, M. Stern, X. Zhou, C. C. Lo, C. D. Weis, T. Schenkel, M. L. W. Thewalt, D. Vion, D. Esteve, B. Julsgaard, K. Moelmer, J. J. L. Morton, and P. Bertet, Reaching the quantum limit of sensitivity in electron spin resonance, Nat. Nanotechnol. 11, 10 (2015).

[15] Y. Kubo, C. Grezes, A. Dewes, T. Umeda, J. Isoya, H. Sumiya, N. Morishita, H. Abe, S. Onoda, T. Ohshima, V. Jacques, A. Dréau, J. F. Roch, I. Diniz, A. Auffeves, D. Vion, D. Esteve, and P. Bertet, Hybrid Quantum Circuit with a Superconducting Qubit Coupled to a Spin Ensemble, Phys. Rev. Lett. 107, 220501 (2011).

[16] W. G. Farr, M. Goryachev, J.-M. le Floch, P. Bushev, and M. E. Tobar, Evidence of dilute ferromagnetism in rareearth doped yttrium aluminium garnet, Appl. Phys. Lett. 107, 122401 (2015).

[17] S. Probst, H. Rotzinger, S. Wünsch, P. Jung, M. Jerger, M. Siegel, A. V. Ustinov, and P. A. Bushev, Anisotropic Rare-Earth Spin Ensemble Strongly Coupled to a Superconducting Resonator, Phys. Rev. Lett. 110, 1 (2013).

[18] C. W. Zollitsch, K. Mueller, D. P. Franke, S. T. B. Goennenwein, M. S. Brandt, R. Gross, and H. Huebl, High cooperativity coupling between a phosphorus donor spin ensemble and a superconducting microwave resonator, Appl. Phys. Lett. 107, 142105 (2015).

[19] D. I. Schuster, A. P. Sears, E. Ginossar, L. Dicarlo, L. Frunzio, J. J. L. Morton, H. Wu, G. A. D. Briggs, B. B. Buckley, D. D. Awschalom, and R. J. Schoelkopf, HighCooperativity Coupling of Electron-Spin Ensembles to Superconducting Cavities, Phys. Rev. Lett. 105, 140501 (2010).

[20] J. Burnett, L. Faoro, I. Wisby, V. L. Gurtovoi, A. V. Chernykh, G. M. Mikhailov, V. A. Tulin, R. Shaikhaidarov, V. Antonov, P. J. Meeson, A. Y. Tzalenchuk, and T. Lindström, Evidence for interacting two-level systems from the $1 / f$ noise of a superconducting resonator, Nat. Commun. 5, 4119 (2014).

[21] W. D. Oliver and P. B. Welander, Materials in superconducting quantum bits, MRS Bull. 38, 816 (2013). 
[22] T. Lindström, J. E. Healey, M. S. Colclough, C. M. Muirhead, and A. Y. Tzalenchuk, Properties of superconducting planar resonators at millikelvin temperatures, Phys. Rev. B - Condens. Matter Mater. Phys. 80, 2 (2009).

[23] Z. Shoudu, W. Siting, S. Xingda, W. Haobing, Z. Heyu, Z. Shunxing, and X. Jun, Czochralski growth of rare-earth orthosilicates $\mathrm{Y}_{2} \mathrm{SiO}_{5}$ single crystals, J. Cryst. Growth 197, 901 (1999).

[24] J. Zmuidzinas, Superconducting microresonators: Physics and applications, Ann. Rev. Condens. Matter Phys. 3, 169 (2012).

[25] A. Bärnthaler, S. Rotter, F. Libisch, J. Burgdörfer, S. Gehler, U. Kuhl, and H. J. Stöckmann, Probing Decoherence Through Fano Resonances, Phys. Rev. Lett. 105, 1 (2010).

[26] D. P. Pappas, M. R. Vissers, D. S. Wisbey, J. S. Kline, and J. Gao, Two level system loss in superconducting microwave resonators, IEEE Trans. Appl. Supercond. 21, 871 (2011).

[27] L. Faoro and L. B. Ioffe, Quantum Two Level Systems and Kondo-Like Traps as Possible Sources of Decoherence in Superconducting Qubits, Phys. Rev. Lett. 96, 1 (2006).

[28] H. Maier-Flaig, Master's thesis, Karlsrhue Institute of Technology, 2013.
[29] I. S. Wisby, S. E. de Graaf, R. Gwilliam, A. Adamyan, S. E. Kubatkin, P. J. Meeson, A. Y. Tzalenchuk, and T. Lindström, Angle-Dependent Microresonator ESR Characterization of Locally Doped $\mathrm{Gd} 3+$ : $\mathrm{Al}_{2} \mathrm{O}_{3}$, Phys. Rev. Appl. 6, 024021 (2016).

[30] E. Abe, H. Wu, A. Ardavan, and J. J. L. Morton, Electron spin ensemble strongly coupled to a three-dimensional microwave cavity, Appl. Phys. Lett. 98, 3 (2011).

[31] A. Bienfait, J. J. Pla, Y. Kubo, X. Zhou, M. Stern, C. C. Lo, C. D. Weis, T. Schenkel, D. Vion, D. Esteve, J. J. L. Morton, and P. Bertet, Controlling spin relaxation with a cavity, Nature 531, 74 (2016).

[32] A. Ortu, A. Tiranov, S. Welinski, F. Fröwis, N. Gisin, A. Ferrier, P. Goldner, and M. Afzelius, Simultaneous coherence enhancement of optical and microwave transitions in solid-state electronic spins, Nat. Mater. 17, 671 (2018).

[33] N. Kukharchyk, S. Pal, J. Rödiger, A. Ludwig, S. Probst, A. V. Ustinov, P. Bushev, and A. D. Wieck, Photoluminescence of focused ion beam implanted $\mathrm{Er} 3+: \mathrm{Y}_{2} \mathrm{SiO}_{5}$ crystals, Phys. Status Solidi (RRL) - Rapid Res. Lett. 8, 880 (2014).

[34] S. Welinski, A. Ferrier, M. Afzelius, and P. Goldner, Highresolution optical spectroscopy and magnetic properties of $\mathrm{Yb}^{3+}$ in $\mathrm{Y}_{2} \mathrm{SiO}_{5}$, Phys. Rev. B 94, 155116 (2016). 